Research

\title{
Genotyping of TRIM5 locus in northern pig-tailed macaques (Macaca leonina), a primate species susceptible to Human Immunodeficiency Virus type I infection Yi-Qun Kuang1,4, Xia Tang1,4, Feng-Liang Liu ${ }^{1,4}$, Xue-Long Jiang2, Ya- Ping Zhang2, Guangxia $\mathrm{Gao}^{3}$ and Yong-Tang Zheng*1
}

Address: ${ }^{1}$ Key Laboratory of Animal Models and Human Disease Mechanisms, Kunming Institute of Zoology, Chinese Academy of Sciences, Kunming, Yunnan 650223, PR China, 2State Key Laboratory of Genetic Resources and Evolution, Kunming Institute of Zoology, Chinese Academy of Sciences, Kunming, Yunnan 650223, PR China, ${ }^{3}$ Institute of Biophysics, Chinese Academy of Sciences, Beijing 100101, PR China and ${ }^{4}$ Graduate School of Chinese Academy of Sciences, Beijing 100039, PR China

Email: Yi-Qun Kuang - kuangyq03@post.kiz.ac.cn; Xia Tang - tangxia123456789@163.com; Feng-Liang Liu - fengliangliu@hotmail.com; XueLong Jiang - jiangxl@mail.kiz.ac.cn; Ya-Ping Zhang - zhangyp@ mail.kiz.ac.cn; Guangxia Gao - gaogx@moon.ibp.ac.cn; Yong-

Tang Zheng* - zhengyt@mail.kiz.ac.cn

* Corresponding author

Published: 9 June 2009

Retrovirology 2009, 6:58 doi:10.1186/1742-4690-6-58

This article is available from: http://www.retrovirology.com/content/6/1/58

(c) 2009 Kuang et al; licensee BioMed Central Ltd.

This is an Open Access article distributed under the terms of the Creative Commons Attribution License (http://creativecommons.org/licenses/by/2.0), which permits unrestricted use, distribution, and reproduction in any medium, provided the original work is properly cited.

\section{Abstract}

Background: The pig-tailed macaques are the only Old World monkeys known to be susceptible to human immunodeficiency virus type I (HIV-I) infection. We have previously reported that the TRIM5-Cyclophilin A (TRIMCyp) fusion in pig-tailed macaques (Macaca nemestrina) is dysfunctional in restricting HIV-I, which may explain why pig-tailed macaques are susceptible to HIV-I infection. Similar results have also been reported by other groups. However, according to the current primate taxonomy, the previously reported $M$. nemestrina are further classified into three species, which all belong to the Macaca spp. This calls for the need to look into the previous studies in more details.

Results: The local species Northern pig-tailed macaque ( $M$. leonina) was analyzed for the correlation of TRIM5 structure and HIV-I infection. Eleven $M$. leonina animals were analyzed, and all of them were found to possess TRIM5-CypA fusion at the TRIM5 locus. The transcripts encoding the dysfunctional TRIM5-CypA should result from the G-to-T mutation in the 3'-splicing site of intron 6. Polymorphism in the putative TRIMCyp recognition domain was observed. The peripheral blood mononuclear cells (PBMCs) of M. leonina were susceptible to HIV-I infection. Consistent with the previous results, expression of the M. leonina TRIMCyp in HeLa-T4 cells rendered the cells resistant to HIV-2 $2_{\text {ROD }}$ but not to SIVmac239 infection.

Conclusion: The susceptibility of $M$. leonina to HIV-I infection is due to the dysfunctional TRIM5CypA fusion in the TRIM5 locus. This finding should broaden our perspective in developing better HIV/AIDS non-human primate animal models. 


\section{Background}

Human immunodeficiency virus type 1 (HIV-1) originated from cross-species transmission from chimpanzees to humans and is the major causative agent of human acquired immunodeficiency syndrome (AIDS) pandemic [1-3]. Although HIV-1 infects human $\mathrm{CD}^{+}$cells, it does not infect most non-human primates (NHP). Studies using Vesicular Stomatitis virus G-glycoprotein (VSV-G) pseudotyped viruses, which bypass the receptor restriction, revealed that species-specific host factors restrict HIV-1 infection [4]. For example, the host restriction factor tripartite motif protein $5 \alpha$ (TRIM $5 \alpha$ ) potently blocks HIV-1 replication in rhesus macaque (M. mulatta) through species-specific post-entry restriction in Old World monkeys [5].

TRIM $5 \alpha$ is a member of the TRIM family, which contains the RING, B-Box 2 and coiled-coil domains, and a C-terminal B30.2/SPRY domain. TRIM5 $\alpha$ interacts with the capsid (CA) portion of HIV-1 Gag protein through its B30.2/ SPRY domain, which determines the specificity and potency of TRIM $5 \alpha$ restriction to retroviruses $[5,6]$. Host protein cyclophilin A (CypA) interacts with the CA through incorporation into HIV-1 particles, and modulates HIV-1 replication in host cells [7-9]. It has been documented that in Old World monkey cells, CypA is required for TRIM5 $\alpha$-mediated resistance to HIV-1 [10]. The New World primate owl monkey (Aotus) expresses a TRIM5-CypA (TRIMCyp) fusion protein, in which the B30.2/SPRY domain of TRIM5 $\alpha$ is replaced by CypA resulting from retrotransposition of the CypA pseudogene cDNA into the seventh intron at the TRIM5 locus. The owl monkey TRIM5-CypA (omTRIMCyp) restricts several retroviruses including HIV-1, simian immunodeficiency virus (SIV) and feline immunodeficiency virus (FIV) $[11,12]$. Recently, we and others reported that in pigtailed macaques the B30.2/SPRY domain is replaced by retrotransposed CypA in the 3'-UTR of TRIM5 in a fashion different from that in the owl monkey, resulting in the failure of restriction to HIV-1 replication in pig-tailed macaques [13-17].

According to the current widely-accepted primate taxonomy based on more morphological studies and phylogeographic analyses, the previously reported Macaca nemestrina group is divided into three species: Sunda pigtailed macaque (M. nemestrina), Northern pig-tailed macaque (M. leonina), and Mentawai macaque (M. pagensis) [18-21]. The M. nemestrina distributes in Malay Peninsula from about $7^{\circ} 30^{\prime} \mathrm{N}$, Sumatra, Bangka and Borneo. The M. leonina ranges from about $8^{\circ} \mathrm{N}$ in Peninsular Thailand, through Burma and Indochina into Bangladesh, India extending north as far as to the Brahmaputra, and the southernmost Yunnan, China. The M. pagensis locates in the Mentawai islands [18]. The previously studied pigtailed macaques may contain individuals of different species. Here, we analyzed the susceptibility of the local species M. leonina in Yunnan to HIV-1 infection and the TRIM5 locus. The fusion pattern of TRIMCyp and the polymorphism of the TRIMCyp recognition domain in $M$. leonina were characterized.

\section{Results \\ Characterization of the TRIMCyp fusion gene in M. leonina}

To investigate the correlation between the TRIM5 $\alpha$ sequence and the susceptibility to infection by HIV-1 in M. leonina, the genomic sequence of the TRIM5 locus of 11 animals from several different populations was analyzed (Table 1). A pair of specific PCR primers was designed based on the human TRIM5 genomic sequence, with the forward primer in the TRIM5 exon 8 and the reverse primer in the adjacent genomic region after TRIM5 3'-UTR (Table 2). A fragment of about 2, 800 bp was amplified (Fig. 1A), indicating that the TRIM5 locus is longer than normal and thus the TRIM5-CypA pattern might exist. To confirm this notion, another pair of primers was designed, with the forward one in exon 8 and the

Table I: The information of $M$. leonina samples used in this study.

\begin{tabular}{|c|c|c|c|c|c|}
\hline Sample Number \# & Sex & Weight & Origin of Macaque & Sampling Time & Population Location \\
\hline 524 & Male & ND & Yunnan, China & $1998-4-15$ & $\mathrm{KIZ}, \mathrm{CAS}$ \\
\hline 528 & Female & ND & Yunnan, China & 1988-4-5 & $\mathrm{KIZ}$, CAS \\
\hline 551 & Female & ND & Yunnan, China & 2000-II & $\mathrm{KIZ}$, CAS \\
\hline 87015 & Male & $\mathrm{l} / \mathrm{kg}$ & Yunnan, China & 2008-4-II & $\mathrm{KIZ}$, CAS \\
\hline 93201 & Male & $12 \mathrm{~kg}$ & Yunnan, China & 2008-4-II & KIZ, CAS \\
\hline 97203 & Male & $9.5 \mathrm{~kg}$ & Yunnan, China & 2008-4-II & KIZ, CAS \\
\hline 99201 & Male & $10 \mathrm{~kg}$ & Yunnan, China & 2008-4-II & KIZ, CAS \\
\hline KMZ-I & Male & $14.5 \mathrm{~kg}$ & Yunnan, China & $2008-3-28$ & KMZ \\
\hline KMZ-2 & Male & ND & Yunnan, China & $2008-3-28$ & $\mathrm{KMZ}$ \\
\hline KMZ-4 & Female & $6.4 \mathrm{~kg}$ & Yunnan, China & $2008-3-28$ & $\mathrm{KMZ}$ \\
\hline KMZ-5 & Male & ND & Yunnan, China & $2008-3-28$ & $\mathrm{KMZ}$ \\
\hline
\end{tabular}

ND: not determined 
Table 2: Primers used for genomic and RT-PCR amplification.

\begin{tabular}{ccc}
\hline No. & Primer $\mathbf{N a m e}$ & Sequence (5' - 3') \\
\hline 1 & T5in6FI & TGGAATTCATGTGGTGTCAGGGTG \\
2 & T5in7FI & CAGCTACCCTGTGGCTTATCAT \\
3 & T5in7RI & GACTTGAGAGAAAGCTGGGAGGA \\
4 & T5ex8FI & CTGGCTCCAAACAACATTTC \\
5 & T5ex8F2 & TGACTCTGTGCTCACCAAGCT \\
6 & T5ex8R I & ATATATAGAAGGCAGAATTGAAG \\
7 & T5ex8R2 & TCAAGAGCTTGGTGAGC \\
8 & T5ex8R3 & AGCCCAGGACGCCAGTACAATA \\
9 & CypAR & TTATTCGAGTTGTCCAC \\
10 & TRIMCypF & ATGGCTTCTGGAATCCTGGTTAATGTAAAG \\
11 & TRIMCypR & CTATTCGAGTTGTCCACAGTCAGCAAT \\
\hline
\end{tabular}

reverse one in the CypA sequence (Table 2). Indeed, a CypA cDNA sequence is inserted in the TRIM5 locus in all M. leonina (Fig. 1B, C), which disrupts the normal TRIM5.

To further characterize the CypA insertion in the TRIM5 locus, we performed several other PCR reactions with different pairs of primers (Table 2). The PCR products were recovered and sequenced. The sequencing results revealed that the CypA pseudogene cDNA insertion in the TRIM5 locus resulted from a LINE (long interspersed nuclear element)-1-mediated retrotransposition (Fig. 1D), which is very common in mammals $[22,23]$.

\section{Expression of TRIMCyp fusion gene in M. leonina}

To test whether the TRIMCyp fusion gene in M. leonina is transcribed to produce mature transcripts, the RNAs from $8 \mathrm{M}$. leonina samples were reverse transcribed and PCR amplified using specific primers TRIMCypF and TRIMCypR (Table 2). Multiple mature TRIMCyp transcripts with different lengths were detected in eight M. leonina samples, but not in the Chinese rhesus macaque samples (data not shown). Sequencing analysis of the PCR products revealed that both exon 7 and exon 8 were spliced out in all major isoforms leaving exon 6 fused to the CypA cDNA in frame (data not shown), as previously reported [13-16].

To understand why exons 7 and 8 were not included in the mature transcripts, the sequences of introns 6 and 7 were analyzed for aberrant splicing sites. The Nsi I restriction site upstream the 3' splicing site of intron 6 has been reported to be closely linked to the mutation within the site [17], which allowed a convenient screening of the Gto-T substitution in the splicing site (Fig. 2B). Analysis of the PCR product flanking intron 6 by the Nsi I restriction digestion revealed that all $M$. leonina were homozygous for the Nsi I site (Fig. 2A). The G-to-T substitution in the 3 ' splicing site of intron 6 was confirmed by sequencing analysis of the PCR products in all the M. leonina samples (Fig. 2B). The G-to-T substitution in the 3 ' splicing site of intron 6 should prevent the inclusion of exon 7 during splicing. Sequencing analysis revealed that the 3' splicing site of intron 7 was normal (data not shown). The exclusion of exon 8 in the mature transcript is likely the result of alternative splicing, as previously observed [13].

\section{Polymorphism analysis in the TRIMCyp recognition domain}

A fragment of 3348 bp from intron 6 to the $3^{\prime}$ genomic adjacent region was analyzed for polymorphisms via DnaSP 4.5 program [24,25]. In the 11 Northern pig-tailed macaques, a total of 46 polymorphic nucleotide sites were identified, including 40 Singleton variable sites and 6 Parsimony informative sites (Fig. 3A). Among these sites, 16 sites (site 579, 592, 613, 796, 803, 883, 925, 1026, 1087, $2134,2251,2303,2415,2494,2506$ and 2529) are in the coding region (39\%), and the others (site 169, 248, 252, $350,364,462,1262,1265,1440,1718,1719,1721$, 1723, 1759, 1765, 1912, 2034, 2037, 2057, 2075, 2740, 2749, 2802, 2868, 2907, 2950, 3016, 3031, 3277 and 3282 ) are in the noncoding region. There are 15 nonsynonymous variation sites in the coding region, except for the synonymous site 2303. In KMZ-4, we identified an insertion-mutation, which results in frame shift relative to the coding region (data no shown). Next, we sought to determine whether the observed polymorphisms are consonant with the real status. Linkage disequilibrium (LD) describes a situation in which some combinations of alleles or genetic markers occur more or less frequently in a population than would be expected from a random formation of haplotypes from alleles based on their frequencies. The occurrence of LD permits the construction of Haplotype. The LD analysis (Fisher's exact test and Chisquare test) showed that no recombinant event occurred, and the degree of LD is strong $(\mathrm{P}<0.001)$ (Fig. 3B). Additionally, the Neutrality theory was used to detect the intraspecific polymorphism level, and two approaches (Tajama's D test and Fu and Li's D test) based on different algorithm models were employed. The Neutrality test of Tajama's D test demonstrated no statistical significance in 
A

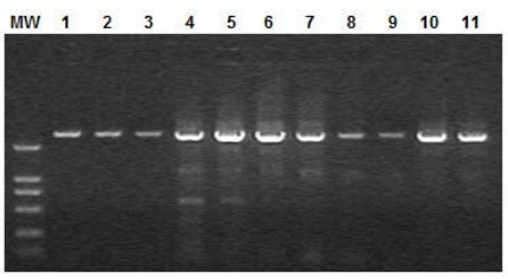

B

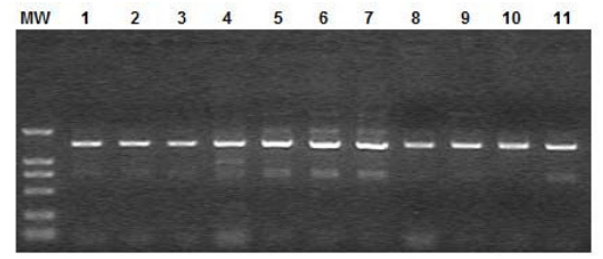

$\mathrm{C}$

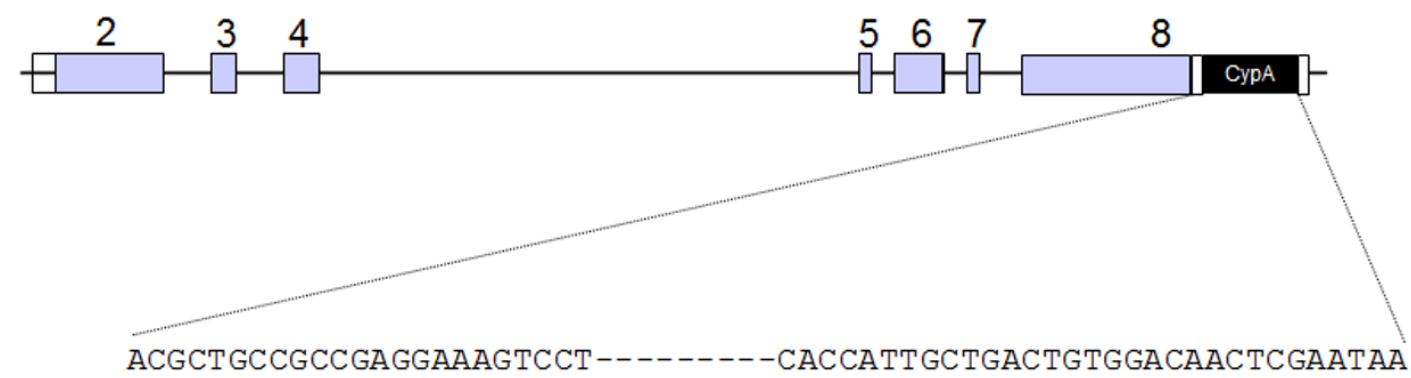

$\mathrm{D}$

......cggggtttccccatggttaggctcgtctagaactcctgacctcaggtgatccacccgcctcggcctgcc aaagtgctgggattacaggcatgagctaccgcgcccagcctgtgcttattttcttaaataatttttgtgg ctttgcag ${ }^{*}$ / ACGCTGCCGCCGAGGAAAGTCCTGTACTACTAGCCATGGTCAACCCTACCGTGTTCTTCGAC ATTGCCGTCGACGGCGAGCCCTTGGGCCGCGTCTCCTTCGAGCTGTTTGCAGACAAGGTTCCAAAGACAGC AGAAAATTTTCGTGCTCTGAGCACTGGAGAGAAAGGATTTGGTTATAAGGGCTCCTGCTTTCACAGAATTA TTCCAGGGTTTATGTGTCAGGGTGGTAACTTCACACACCATAATGGCACTGGTGGCAAGTCCATCTATGGG GAGAAATTTGAAGATGAGAACTTCATCCTAAAGCATACAGGTCCTGGCATCTTGTCCATGGCAAATGCTGG ACCCAACACAAATGGTTCCCAGTTTTTCATCTGCACTGCCAAGACTGAGTGGTTGGATGGCAAGCATGTGG TCTTTGGCAAAGTGAAAGAAGGCATGAATATTGTGGAGGCCATGGAGCGCTTTGGGTCCAGGAATGGCAAG ACCAGCAAGAAGATCACCATTGCTGACTGTGGACAACTCGAATAAAATCGTCGAACGGCAGGCGTGCAAAC TTGGCGTAATCATGGACAACTCGAATAA.......ATAAAAACTAAGTAACAATTAaaaaataataatatatt tttgtattaaaaa......

\section{Figure I}

Formation of TRIMCyp fusion gene in M. leonina. (A and B) The genomic sequences spanning from the 5 ' end of exon 8 to the 3' end of exon 8 (A) or to the 3'-UTR of CypA cDNA (B) were PCR amplified and subject to electrophoresis analysis. MW: DNA molecular weight marker DL-2000; Lane I-II: M. leonina samples 524, 528, 55I, KMZ-I, KMZ-2, KMZ-4, KMZ-5, 870I5, 9320I, 97203, and 9920I. (C) Schematic structure of the TRIM5Cyp fusion. The exons are represented as boxes with the coding region being shaded, and the sequence of the inserted CypA cDNA is denoted below. (D) The CypA pseudogene cDNA retrotransposed into TRIM5 locus. The asterisk $(*)$ indicates the splicing acceptor, CypA pseudogene cDNA sequence is underlined, target site duplication (TSD) is in bold italic, and the start or stop codon of inserted CypA CDNA is in bold-type. 
A

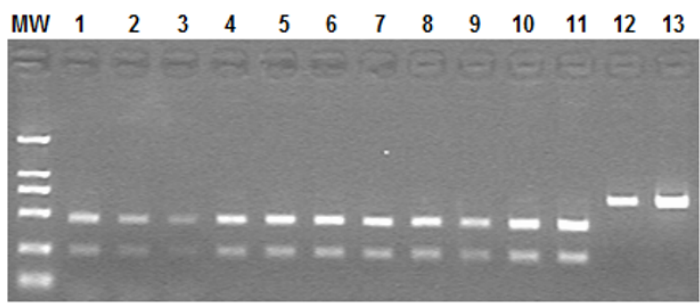

B

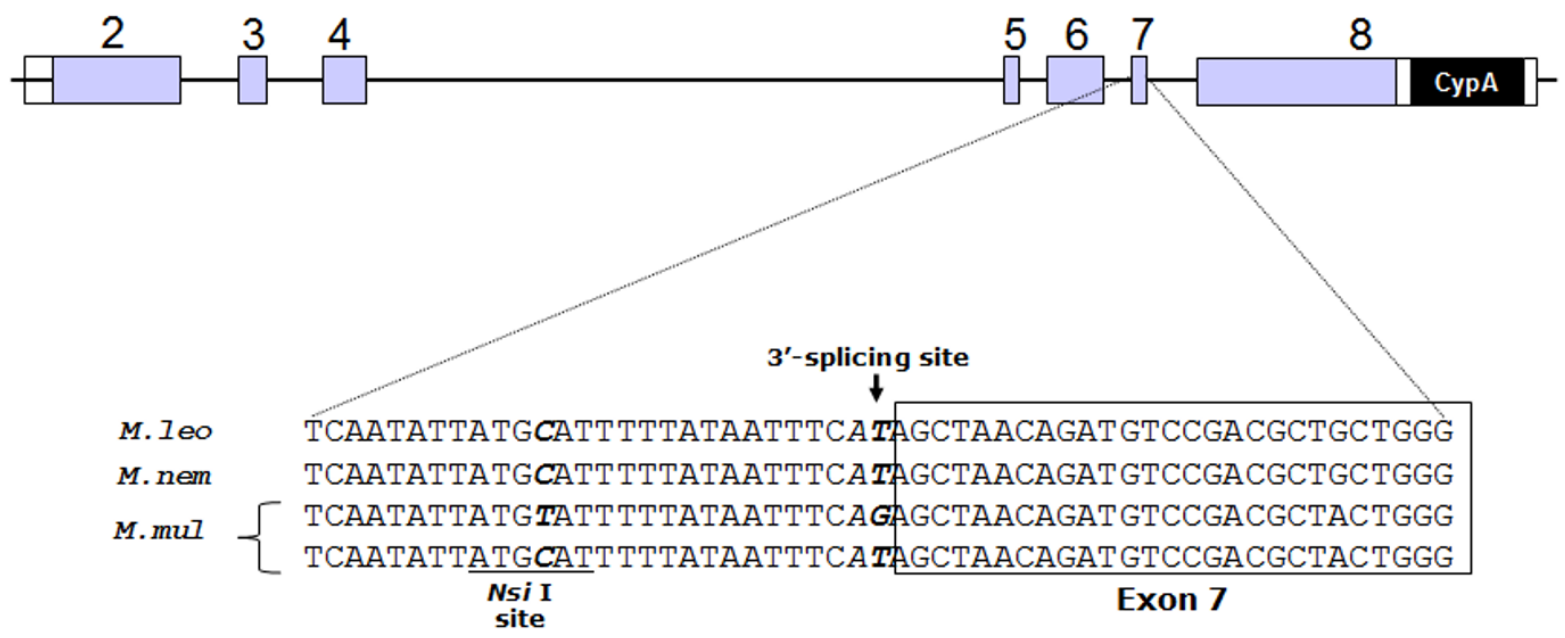

\section{Figure 2}

Analysis of the 3'-splicing site in intron 6 at the TRIM5 locus. (A) The sequences encompassing the 3 '-splicing site in intron 6 were PCR amplified from the genomic DNA of the following samples. The PCR products were digested with restriction endonuclease Nsi I, followed by electrophoresis in a I.4\% agarose gel. MW: molecular weight DNA marker DL-2000; Lane I-I I: M. leonina samples as described in the legend to figure IB; Lane I2: M. mulatta 95005 PBMCs; Lane I3: a M. mulatta immortalized B cell line. (B) Schematic representation of the position and sequences of the 3'-splicing acceptor site in intron 6. The boxes represent the exons of the TRIM5 genome, and the lines represent the introns. The 3'-splicing site is indicated by the arrow, and the Nsi I recognition sequence is underlined.

polymorphism along the sequences $(\mathrm{P}<0.005)$, while $\mathrm{Fu}$ and Li's D test were significant $(\mathrm{P}<0.005)$ (Fig. 3C).

The coding sequences of TRIMCyp exon 7, exon 8 and CypA from M. leonina were assembled to deduce the putative amino acid sequences. The phylogenetic tree based on the putative amino acid sequences demonstrated that the 11 M. leonina are divided into several major subgroups (Fig. 4A), which may explain the high polymorphic in this region. In the Aotus and Macaca TRIMCyp proteins, the CypA part is the recognition domain mediating the binding of the fusion protein to the incoming viral capsids. The putative amino acid sequences of the CypA domain from various species were aligned. No major differences were found in sequences of CypA inserted in TRIMCyp from diverse M leonina. The results clearly show that the sequences are homologous among the old World macaque M. leonina, M. nemestrina and M. mullata species, while the M. fasciculari and the New World monkey A. trivirgatus were much less homologous (Fig. 4B). In addition, some amino acids critical for the restriction of HIV1 in A. trivirgatus, such as N66 and H69, were observed in M. leonina. The phylogenetic tree among these primates was constructed based on the inserted CypA amino acid sequences. The result revealed that the Macaca spp species members M. leonina, $M$. nemestrina and $M$. mulatta were 
A

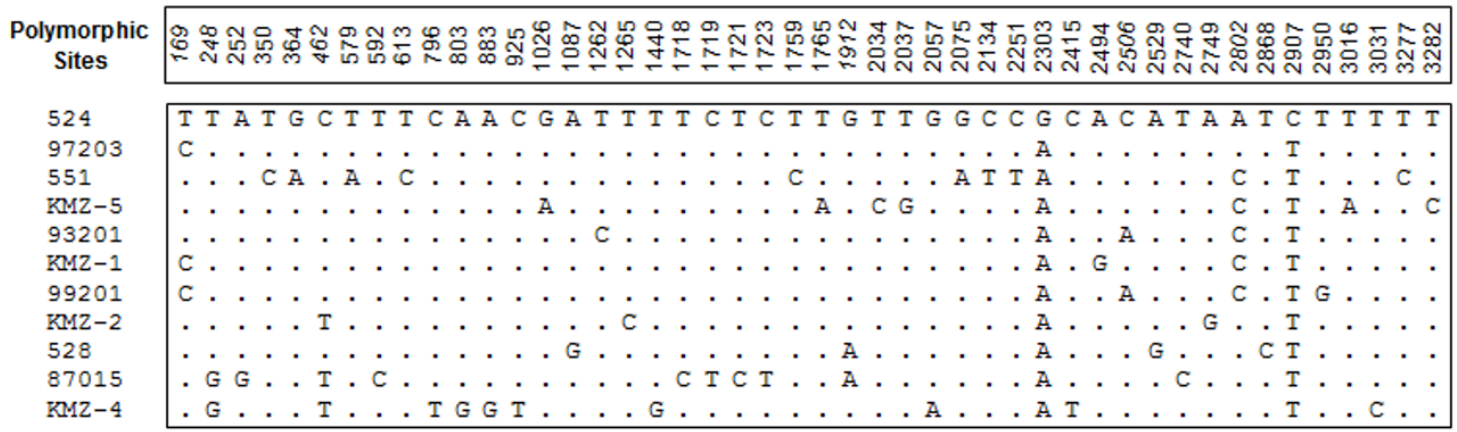

B

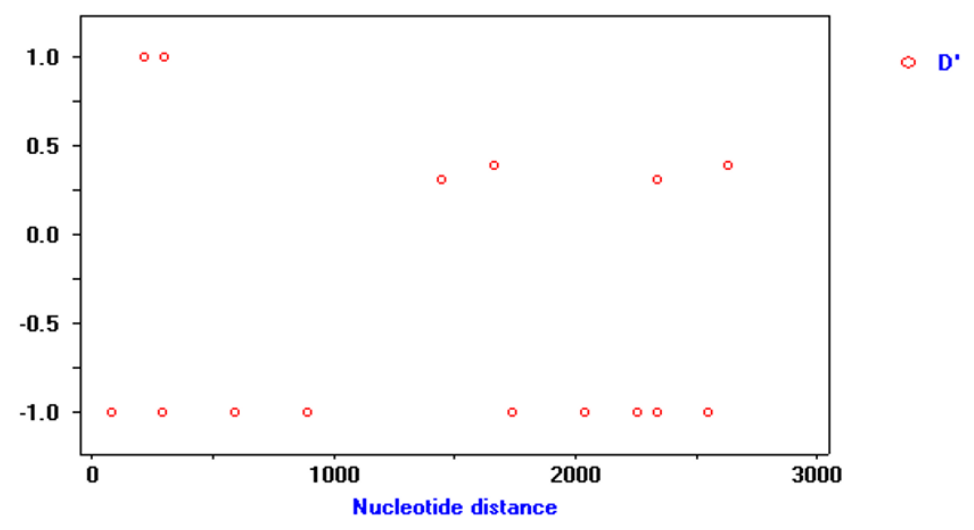

C

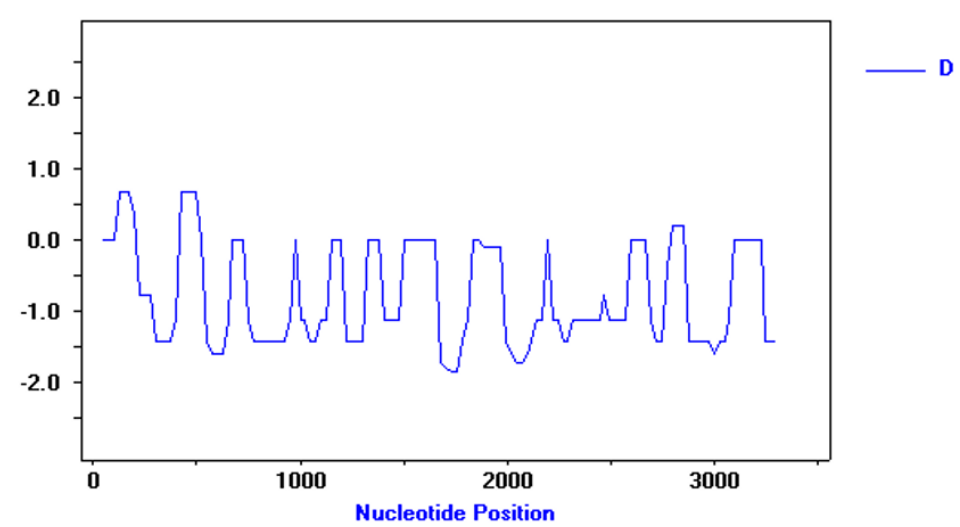

\section{Figure 3}

Genetic polymorphism analysis of the TRIMCyp recognition domain. (A) Polymorphic sites of the nucleotide sequence of the TRIMCyp recognition domain. The boxed numbers indicate the polymorphic sites, with 6 Parsimony informative positions in italic. The boxed sequences showed the Haplotype of the $M$. leonina samples. Dots present the identical nucleotides. (B) The linkage disequilibrium analysis of all sequenced sites. The histogram $X$ axis plots the $D^{\prime}$ value, the $Y$ axis plots the DNA sequence nucleotide positions. (C) Tajima's or Fu and Li's D test of the total number of mutations for neutrality test through DnaSP program. 
A

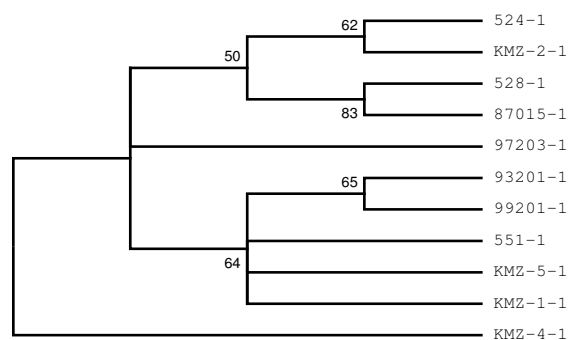

B
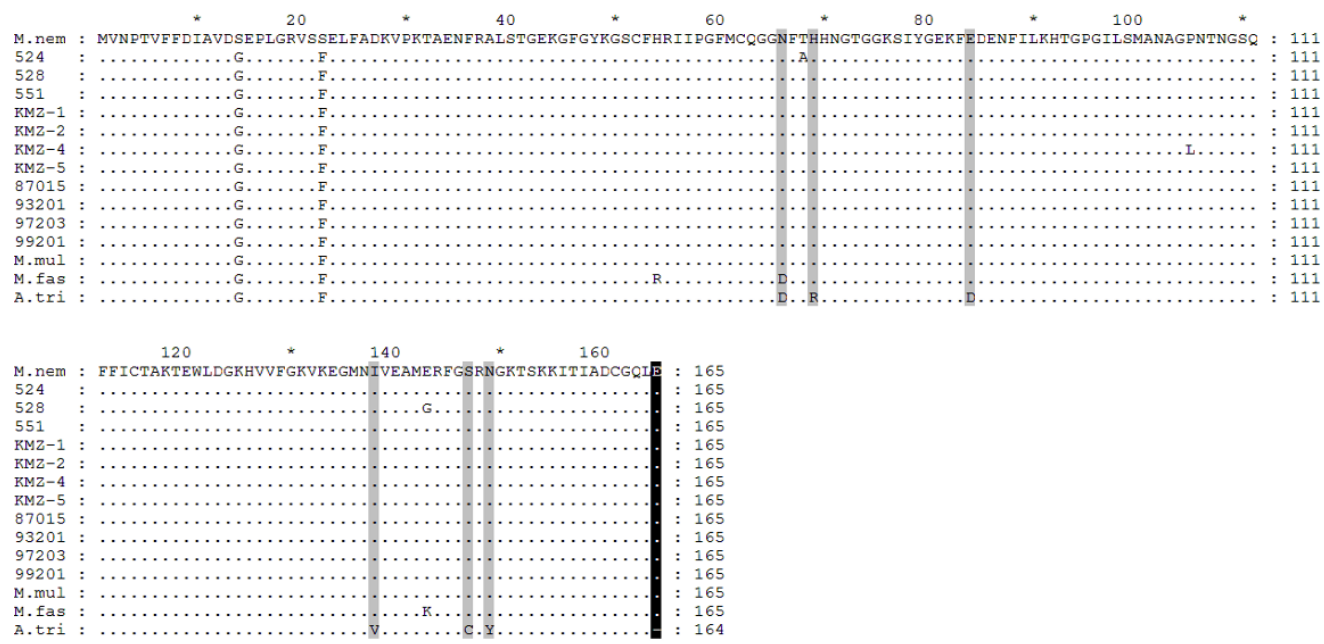

$\mathrm{C}$

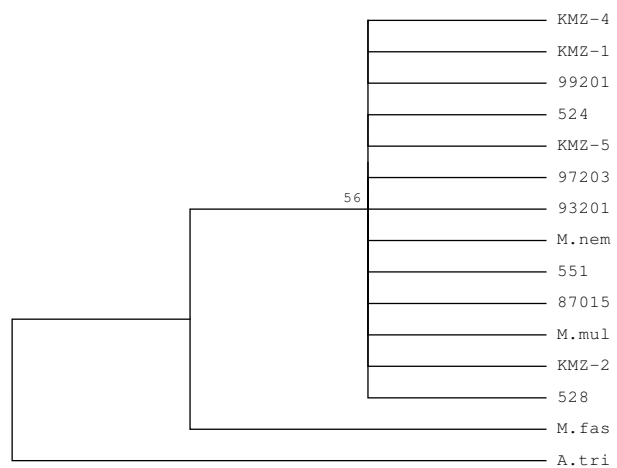

Figure 4

Phylogenetic analysis of primate TRIMCyp recognition domain. (A) Neighbor-joining nucleotide tree of the II Northern pig-tailed macaques based on TRIMCyp. Bootstrap values are based on I,000 replicates. (B) Alignment of putative CypA amino acid sequences of primate TRIMCyp. The gray bar indicates mutation sites in Old World primates, and the bold bar indicates deletion mutations, as compared to $A$. trivirgatus. (C) Phylogenetic analysis of the primate TRIMCyp recognition domain (based on CypA amino acid sequence) with the A. trivirgatus as outgroup. Bootstrap values are based on I,000 replicates. 
relatively close in homology, the $M$. fasciculari and the New World monkey A. trivirgatus were in two obviously more distant groups (Fig. 4C).

\section{Susceptibility of M. leonina PBMCs to HIV-I infection}

To determine the susceptibility of $M$. leonina to infection by HIV-1, the PBMCs were isolated from EDTA K2-treated whole blood. The PBMCs from M. mulatta, which are known to be resistant to HIV-1 infection, were used as a negative control, and the PBMCs from human, which are known to be susceptible to HIV-1 infection, were used as a positive control. The susceptible human T cell line MT4 , the monocyte cell line U937, and the resistant Chinese rhesus macaque transformed B lymphocyte cell line were also used as controls. The cells were challenged with VSVG pseudotyped HIV-1 carrying the GFP reporter (HIVGFP-VSVG) at the infection unit (IU) of 0.1 and 0.5 . The percentage of GFP positive cells counted by FACS analysis was used as an indicator for the susceptibility of the cells to HIV-1 infection. As expected, the M. mulatta PBMCs were relatively resistant to HIV-1 infection (Fig. 5A). In comparison, the M. leonina PBMCs demonstrated a magnitude of susceptibility to HIV-GFP-VSVG infection comparable to the human PBMCs (Fig. 5A). These results established that the M. leonina are susceptible to HIV-1 infection.

To test whether the M. leonina TRIMCyp can restrict other retroviruses, we generated HeLa-T4 cell lines expressing Cterminally HA-tagged TRIMCyp of M. leonina and A. trivirgatus. The expression of the TRIMCyp fusion proteins (npmTRIMCyp for northern pigtailed macaque TRIMCyp and omTRIMCyp for owl monkey TRIMCyp) was confirmed by Western immunoblotting (Fig. 5B). The cells were challenged with $\mathrm{HIV}-2_{\mathrm{ROD}}(\mathrm{MOI}=0.02)$. Replication of the virus in these cells was evaluated by measuring the capsid p27 antigen levels. The results demonstrated that both npmTRIMCyp and omTRIMCyp actively restricted HIV-2 $2_{\text {ROD }}$ by about 16-fold (Fig. 5C). The cells were also assayed for their restriction to SIVmac239 replication. The npmTRIMCyp demonstrated very moderate restriction activity, while the omTRIMCyp could not inhibit SIVmac239 replication (Fig. 5C).

\section{Discussion}

Because of their close evolutionary relationship to humans, NHPs are of vital importance in biomedical research and are often the best or only animal models for controlled experiments relevant to a range of human diseases and disorders. Macaque infection models provide unique opportunities for generating discoveries that may lead to new therapeutic options, improved vaccine strategies, and increased preparedness for future disease outbreaks. Among Old World monkeys, the pig-tailed macaques ( $M$. nemestrina) were reported to be prone to
HIV-1 infection with AIDS-like symptoms [26-29]. We and other groups identified TRIMCyp fusion protein expression in pig-tailed macaques. However, the fusion protein failed to restrict HIV-1 replication when expressed in some non-restrictive human or macaque cell lines [1317].

Interestingly, the aforementioned pig-tailed macaques are divided into three macaque species according to current primate taxonomy [18]. Understanding the relationship between the TRIM5 locus and the susceptibility to HIV-1 in these species is urgently required. Here, we surveyed the M. leonina in Yunnan province, China. The results showed that the PBMCs of M. leonina are susceptible to HIV-1 infection, a finding which is consistent with previous results [13-17]. Moreover, the M. leonina fusion protein npmTRIMCyp can potently block HIV-2 $2_{\mathrm{ROD}^{\prime}}$ which may account for the different modulatory roles of host cell CypA, as the CypA is incorporated into HIV-1 but not HIV-2. Some other mechanisms may also exist, and one needs more work to dissect these possibilities. The same fusion pattern of TRIM5-CypA gene was observed in all the 11 surveyed M. leonina animals. Although it has been reported that both $M$. nemestrina and $M$. mulatta express TRIMCyp fusion proteins [15-17], our results suggest that the frequency of TRIM5-CypA fusion is higher in $M$. leonina than in M. mulatta. Further studies suggest that the fusion results from the insertion of the CypA pseudogene CDNA into the 3'-UTR of TRIM5 through the LINE-1-element-mediated retrotransposition. The transcription products of TRIM5-CypA were also detected in M. leonina PBMCs, and the transcript formation was attributed to the G-to-T substitution in the 3'-splicing site of TRIM5 intron 6 in macaques 17. In the human genome, more than 60 processed CypA pseudogenes were reported across the genome by retrotransposition [30]. In New World primates, the TRIM5-CypA fusion gene was only identified in Aotus species, and the CypA exposed positive selection in the evolutionary history [31]. However, what drove the CypA pseudogene cDNA retrotransposition into the TRIM5 locus twice in the New World and Old World primates independently? These questions call for more genetic research to delineate the mechanistic details.

Host genetic variations have important impact on the susceptibility to HIV-1 infection. TRIM5 $\alpha$ restricts HIV-1 through the B30.2/SPRY domain specifically recognizing and interacting with the CA protein. In owl monkey, the CypA copy in the TRIMCyp fusion protein can bind lentiviral CA and block their replication $[11,12]$. In pig-tailed macaques, CypA pseudogene cDNA substituted the B30.2/ SPRY in the TRIM $5 \alpha$, resulting in the formation of the TRIMCyp fusion gene. Virgen et al. suggested that the TRIMCyp protein from $M$. nemestrina does not bind to HIV-1 CA because of the single amino acid (R69H) muta- 
A

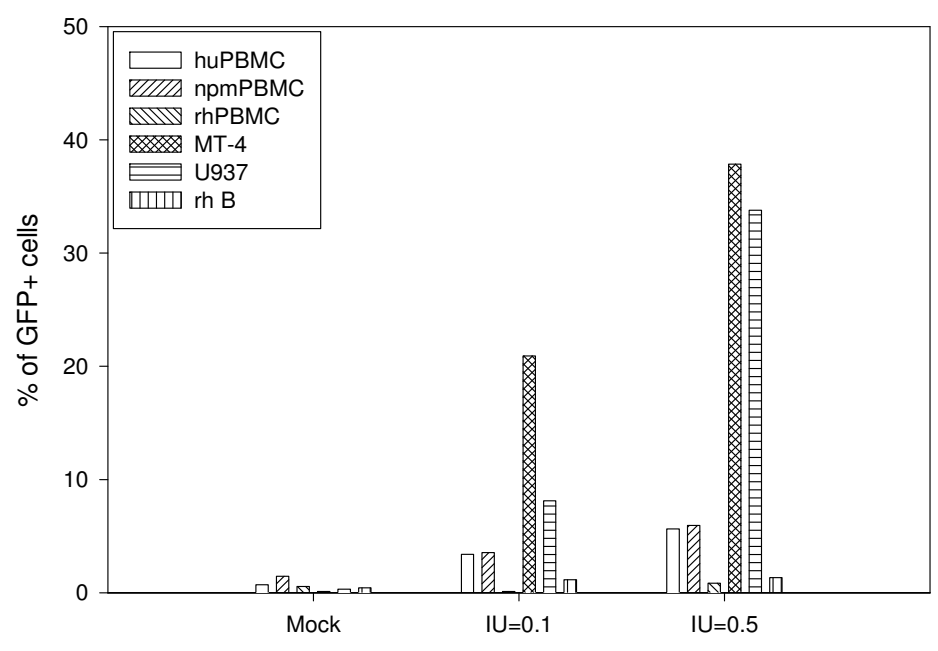

B

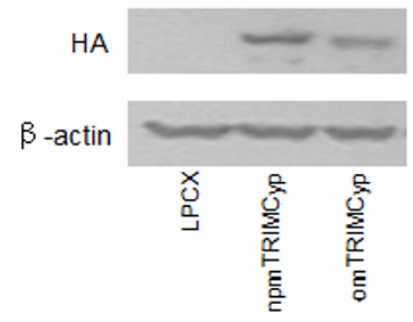

C

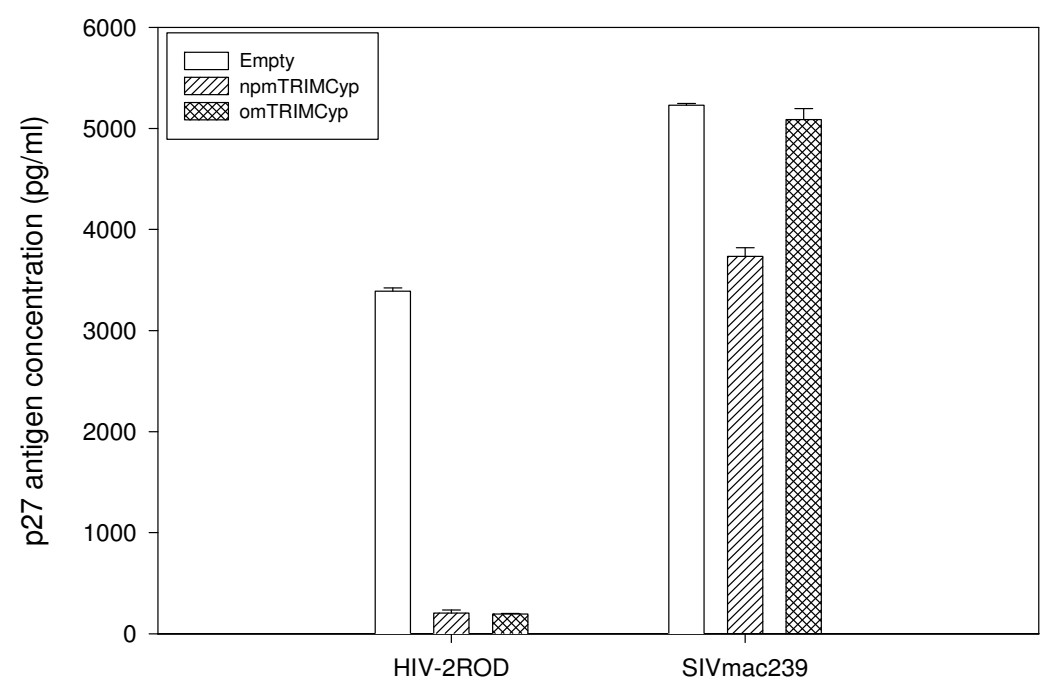

Figure 5 (see legend on next page) 
Figure 5 (see previous page)

Restriction activities of $M$. leonina TRIMCyp on lentiviruses. (A) The indicated cells were infected with HIV-GFP-VSVG at the indicated infection unit (IU). IU $=0.1$ or 0.5 denotes 10 -fold or 2 -fold dilution of HIV-GFP-VSVG viral stocks, respectively. Percentage of infected cells was counted 48 hours later by FACS analysis of GFP positive cells. The result is representative of three independent experiments. nPmPBMC: northern pig-tailed macaque PBMC; huPBMC: human PBMC; rhPBMC: rhesus PBMC; MT-4: human T cell line MT-4; U937: human monocyte line U937; rh B: a Chinese rhesus macaque transformed $B$ immortalized cell line. (B) The HA-tagged TRIMCyp cloned from northern pig-tailed macaque and owl monkey TRIMCyp were stably expressed in HeLa-T4 cells. The expression of the proteins was confirmed by Western blotting. (C) The cells were infected with HIV-2 $2_{\text {ROD }}$ or SIVmac239 virus at MOI (multiplicity of infection) $=0.02$. Four days post-infection, the capsid p27 antigen levels in the culture supernatants were measured by quantitative ELISA assays. The result is representative of three independent experiments.

tion in the TRIMCyp-CA interaction interface [15]. Interestingly, the npmTRIMCyp also contains the $\mathrm{R} 69 \mathrm{H}$ mutation in the CypA domain, which may partly explain why the npmTRIMCyp cannot restrict HIV-1. The recognition domain of M. leonina TRIMCyp is relatively highly polymorphic. It would be interesting to analyze whether the specific polymorphism affects the sensitivity of the primates to HIV-1 infection. It might be possible to establish optimal HIV/AIDS NHP models in M. leonina through screening macaques that possess SNPs more susceptible to HIV-1 infection.

In conclusion, the TRIM5-CypA fusion in M. leonina maybe a pivotal factor associated with their susceptibility to HIV-1. The M. leonina appears to be a good candidate for an HIV/AIDS animal model. Our results should broaden the perspective in developing better HIV/AIDS NHP animal models.

\section{Materials and methods Animals}

Northern pig-tailed macaques KMZ-1, KMZ-2, KMZ -4 and KMZ-5 were bred in the Kunming Zoo (KMZ), animals 524, 528, 551, 87015, 93201, 97203 and 99201 were raised in the Kunming Institute of Zoology (KIZ), Chinese Academy of Sciences (CAS) (Table 1). The Chinese rhesus macaque 95005 was raised in the KIZ, CAS. Whole blood from these animals was collected in EDTAK Blood Collection Tubes following the National Experimental Animal Handling Ordinance.

\section{RNAIDNA samples}

PBMCs were isolated from EDTA $\mathrm{K}_{2}$-treated whole blood by EZ-Sep ${ }^{\mathrm{Tm}}$ Monkey $9 \times$ (Dakewe Biotech) or FicollHypaque density centrifugation. Total RNA was isolated from $8 \times 10^{6}$ PBMCs using the RNAprep Cell Kit (Tiangen) following the manufacturer's instruction. Genomic DNA was isolated from $300 \mu \mathrm{l}$ whole blood using the Puregene DNA Purification Kit (Qiagen) following the manufacturer's handbook.

\section{Genomic DNA Amplification and Sequencing}

The PCR primers for amplifying the genomic DNA from intron 6 to the 3' flanking sequence (Table 2) were designed based on the sequences published in Genbank (accession numbers EU371641 and NT 009237). The fragments were amplified using LATaq-PCR or ExTaq-PCR kit (TaKaRa), purified with DNA Gel Extraction Kit (Watson Biotech), and cloned into the pMD 19-T Simple vector (TaKaRa), clones were picked up for sequencing analysis.

To screen for the G-to-T mutation associated with the 3'splicing site within TRIM5 intron 6 , the fragment encompassing the 3 '-splicing site in intron 6 was PCR amplified with the sense primer T5in6F1 and the anti-sense primer T5ex8R3. The PCR products were digested with restriction endonuclease Nsi I (Fermentas), followed by electrophoresis in a $1.4 \%$ agarose gel [17]. In addition, the PCR products were purified and cloned for sequencing analysis.

\section{cDNA Amplification}

Primers TRIMCypF and TRIMCypR (Table 2) for amplification of the complete coding sequence of Northern pigtailed macaques TRIM5 have been described previously [13]. The total RNA was reverse transcribed into cDNA using the PrimeScript $1^{\text {st }}$ Strand cDNA Synthesis Kit (TaKaRa) following the manufacturer's instruction. The PCR condition was: $94^{\circ} \mathrm{C}$ for 2 minutes; 30 cycles of $94^{\circ} \mathrm{C}$ for 30 seconds, $55^{\circ} \mathrm{C}$ for 30 seconds, and $72^{\circ} \mathrm{C}$ for 1.5 minutes; held at $72^{\circ} \mathrm{C}$ for 7 minutes, and stored at $4^{\circ} \mathrm{C}$.

\section{Sequence analysis}

The sequences were assembled with the Contig program, and aligned by the Clustal X 1.83 or DNAStar 7.1.0 (Lasergene) software. Nucleotide sequence polymorphisms were analyzed by DnaSP 4.50 program. Neighbor-joining nucleotide tree of primate TRIMCyp was constructed via MEGA 3.1 program, bootstrap values were based on 1,000 replicates. Representative species of Aotus trivirgirtas was used as outgroup. The reference sequence accession num- 
bers are: EU371639, EU371641, EU328216, $\underline{\text { AY646199, }}$ EU328216.

\section{Cell culture, transfection, and infection assay}

Primates and human PBMCs were isolated by EZ-Sep monkey $9 \times$ or Ficoll-Hypaque PBMCs separation solution. PBMCs were cultured in RPMI 1640 complete medium supplemented with $5 \mu \mathrm{g} / \mathrm{ml}$ Phytohemagglutinin (PHA) (Sigma-Aldrich) and $50 \mathrm{IU} / \mathrm{ml}$ Interleukin-2 (IL-2) (Sigma-Aldrich) for 72 hours to activate the cells. Human T lymphocyte MT-4, monocyte U937, and a transformed Chinese rhesus macaque B lymphocyte line were cultured in complete RPMI 1640 medium. Activated PBMCs were seeded at the density of $5 \times 10^{5}$ cells/well in the 96-well plate, and the control cell lines were seeded at $4 \times 10^{5}$ cells/well. The HIV-GFP-VSVG viral stocks were thawed in a room temperature water bath, diluted with culture medium supplemented with $20 \mathrm{mM}$ pH7.5 HEPES and $8 \mu \mathrm{g} / \mathrm{ml}$ polybrene (Sigma-Aldrich) on the ice. The cells were infected for 4 hours in the $37^{\circ} \mathrm{C}, 5 \% \mathrm{CO}_{2}$ chamber, washed twice with phosphate-buffered saline (PBS), and then cultured in fresh RPMI 1640 complete medium. After 48 hours post-infection, the percentage of GFP positive cells was counted by FACS analysis using a FACSCalibur (Becton Dickinson).

HeLa-T4 cells were cultured in DMEM supplemented with $10 \%$ fetal calf serum (GIBCO), penicillin (Sigma), and streptomycin (Invitrogen). The C-terminally avian influenza Hemagglutinin (HA)-tagged TRIMCyp cDNA recombinant pLPCX expression plasmids of M. leonina and $A$. trivirgatus were constructed and transfected as previously described [13], npmTRIMCyp and omTRIMCyp expression in HeLa-T4 cells were detected by Western blot for the HA-tag, $\beta$-actin as a loading control. HeLa-T4 cells stably expressing TRIMCyp proteins were seeded in the 24well plate at a density of $3 \times 10^{4}$ cells/well. On the following day, the cells were inoculated with viruses $(\mathrm{MOI}=$ 0.02 ) at $37^{\circ} \mathrm{C}$ for 2 hours. The cells were washed twice with PBS and cultured in $500 \mu \mathrm{l}$ of fresh DMEM complete medium at $37^{\circ} \mathrm{C}, 5 \% \mathrm{CO}_{2}$. Culture supernatants were harvested in duplicate on day 4 post-infection. The levels of HIV-2 $2_{\text {ROD }}$ and SIVmac239 capsid proteins in the medium were quantified by a p27-specific SIV p27 antigen ELISA kit (Zeptometrix).

\section{Nucleotide sequence accession numbers}

The M. leonina TRIM5-CypA sequences are submitted for deposition in the GenBank database, the accession numbers are GQ180913-GQ180923.

\section{Competing interests}

The authors declare that they have no competing interests.

\section{Authors' contributions}

YTZ and YQK conceived of the study, and participated in its design. YQK, XT, and FLL carried out the experiments. YQK, YTZ, GG, XLJ and YPZ analyzed the results and drafted the manuscript. All authors read and approved the final manuscript.

\section{Acknowledgements}

We are grateful to Ms. M. Yang of Kunming Zoo and Mr. Y. Yan of Kunming Primate Research Center for kindly providing Northern pig-tailed macaque blood samples. This study was supported in part by grants to YTZ from NSFC (3067I 960, U083260I), 973 program (2006CB504302,

2006CB504208, 2009CB522306), CAS (KSCXI-YW-R-I5, KSCX2-YW-R092), and Scientific and Technological projects of China (2008ZXI000I002, 2008ZX1000I-0I3, 2008ZXI0005-005) and Yunnan (2006PT08, 2007BC006).

\section{References}

I. Simon $F$, Mauclère $P$, Roques $P$, Loussert-Ajaka I, Müller-Trutwin MC Saragosti S, Georges-Courbot MC, Barré-Sinoussi F, Brun-Vézinet F: Identification of a new human immunodeficiency virus type I distinct from group $\mathbf{M}$ and group $\boldsymbol{O}$. Nat Med 1998, 4(9): 1032-1037.

2. Gao F, Bailes E, Robertson DL, Chen Y, Rodenburg CM, Michael SF, Cummins LB, Arthur LO, Peeters M, Shaw GM, Sharp PM, Hahn BH: Origin of HIV-I in the chimpanzee Pan troglodytes troglodytes. Nature 1999, 397(67| 18):436-44I.

3. Keele BF, Van Heuverswyn F, Li Y, Bailes E, Takehisa J, Santiago ML, Bibollet-Ruche F, Chen Y, Wain LV, Liegeois F, Loul S, Ngole EM, Bienvenue Y, Delaporte E, Brookfield JF, Sharp PM, Shaw GM, Peeters $\mathrm{M}, \mathrm{Hahn} \mathrm{BH}$ : Chimpanzee reservoirs of pandemic and nonpandemic HIV-I. Science 2006, 3 I 3(5786):523-526.

4. Hofmann W, Schubert D, LaBonte J, Munson L, Gibson S, Scammell J, Ferrigno P, Sodroski J: Species-specific, postentry barriers to primate immunodeficiency virus infection. J Virol 1999, 73(12): 10020-10028.

5. Stremlau M, Owens CM, Perron MJ, Kiessling M, Autissier P, Sodroski I: The cytoplasmic body component TRIM5 $\alpha$ restricts HIV-I infection in Old World monkeys. Nature 2004, 427(6977):848-853.

6. Stremlau M, Perron M, Welikala S, Sodroski J: Species-specific variation in the B30.2(SPRY) domain of TRIM5alpha determines the potency of human immunodeficiency virus restriction. J Virol 2005, 79(5):3|39-3।45.

7. Franke EK, Yuan HE, Luban J: Specific incorporation of cyclophilin A into HIV-I virions. Nature 1994, 372(6504):359-362.

8. Thali M, Bukovsky A, Kondo E, Rosenwirth B, Walsh CT, Sodroski J, Göttlinger HG: Functional association of cyclophilin A with HIV-I virions. Nature 1994, 372(6504):363-365.

9. Towers GJ, Hatziioannou T, Cowan S, Goff SP, Luban J, Bieniasz PD: Cyclophilin A modulates the sensitivity of HIV-I to host restriction factors. Nat Med 2003, 9(9): I I38-I I 43

10. Berthoux L, Sebastian S, Sokolskaja E, Luban J: Cyclophilin A is required for TRIM5\{alpha\}-mediated resistance to HIV-I in Old World monkey cells. Proc Natl Acad Sci USA 2005, I02(4I): | 4849-| 4853.

II. Sayah DM, Sokolskaja E, Berthoux L, Luban J: Cyclophilin A retrotransposition into TRIM5 explains owl monkey resistance to HIV-I. Nature 2004, 430(6999):569-573.

12. Nisole S, Lynch C, Stoye JP, Yap MW: A Trim-cyclophilin A fusion protein found in owl monkey kidney cells can restrict HIV-I. Proc Natl Acad Sci USA 2004, 10 I (36): I3324-I 3328.

13. Liao CH, Kuang YQ, Liu HL, Zheng YT, Su B: A novel fusion gene TRIM5-Cyclophilin A in the pig-tailed macaque determines its susceptibility to HIV-I infection. AIDS. 2007, 2 I (SuppI 8):S19-S26.

14. Wilson SJ, Webb BL, Ylinen LM, Verschoor E, Heeney JL, Towers GJ: Independent evolution of an antiviral TRIMCyp in rhesus macaques. Proc Natl Acad Sci USA 2008, 105(9):3557-3562. 
15. Virgen CA, Kratovac Z, Bieniasz PD, Hatziioannou T: Independent genesis of chimeric TRIM5-cyclophilin proteins in two primate species. Proc Natl Acad Sci USA 2008, I05(9):3563-3568.

16. Brennan G, Kozyrev Y, Hu SL: TRIMCyp expression in Old World primates Macaca nemestrina and Macaca fascicularis. Proc Natl Acad Sci USA 2008, I 05(9):3569-3574.

17. Newman RM, Hall L, Kirmaier A, Pozzi LA, Pery E, Farzan M, O'Neil SP, Johnson W: Evolution of a TRIM5-CypA splice isoform in old world monkeys. PLoS Pathog 2008, 4(2): el 000003.

18. Groves C: Primate Taxonomy Washington DC: Smithsonian Institution Press; 200I.

19. Rosenblum LL, Supriatna J, Melnick DJ: Phylogeographic analysis of pigtail macaque populations (Macaca nemestrina) inferred from mitochondrial DNA. Am J Phys Anthropol 1997, I 04( I):35-45.

20. Gippoliti S: Notes on the taxonomy of Macaca nemestrina leonina blyth, I863 (Primates: Cercopithecidae). Hystrix It J Mamm 200I, I 2(I):5I-54.

21. Li QQ, Zhang YP: Phylogenetic relationships of the macaques (Ceropithecidae: Macaca), inferred from Mitochondrial DNA sequences. Biochem Genet 2005, 43(7-8):375-386.

22. Kazazian HH Jr: Mobile elements: Drivers of genome evolution. Science 2004, 303(5664): I626-1632.

23. Vincent BJ, Myers JS, Ho HJ, Kilroy GE, Walker JA, Watkins WS, Jorde LB, Batzer MA: Following the LINEs: An analysis of primate genomic variation at human-specific LINE-I insertion sites. Mol Biol Evol 2003, 20(8): I338-1348.

24. Rozas J, Rozas R: DnaSP version 3: an integrated program for molecular population genetics and molecular evolution analysis. Bioinformatics 1999, I 5(2): I74-I75.

25. Rozas J, Gullaud M, Blandin G, Aguadé M: DNA variation at the rp49 gene region of Drosophila simulans: Evolutionary inferences from an unusual haplotype structure. Genetics 200I I 58(3): I |47-I I55.

26. Agy MB, Frumkin LR, Corey L, Coombs RW, Wolinsky SM, Koehler J, Morton WR, Katze MG: Infection of Macaca nemestrina by human immunodeficiency virus type-I. Science 1992, 257(5066): $103-106$.

27. Kent SJ, Corey L, Agy MB, Morton WR, McElrath MJ, Greenberg PD: Cytotoxic and proliferative $T$ cell responses in HIV-Iinfected Macaca nemestrina. J Clin Invest 1995, 95(I):248-256.

28. Bosh ML, Schmidt A, Chen J, Florey MJ, Agy M, Morton WR Enhanced replication of HIV-I in vivo in pigtailed macaques (Macaca nemestrina). J Med Primatol. 2002, 29(3-4): I07-I I 3.

29. Pekrun K, Shibata R, Igarashi T, Reed M, Sheppard L, Patten PA, Stemmer WP, Martin MA, Soong NW: Evolution of a human immunodeficiency virus type I variant with enhanced replication in pig-tailed macaque cells by DNA shuffling. J Virol 2002, 76(6):2924-2935.

30. Zhang Z, Harrison PM, Liu Y, Gerstein M: Millions of years of evolution preserved: a comprehensive catalog of the processed pseudogenes in the human genome. Genome Res 2003, I 3(I 2):254|-2558.

31. Ribeiro IP, Menezes AN, Moreira MAM, Bonvicino CR, Seuánez HN Soares MA: Evolution of Cyclophilin A and TRIMCyp retrotransposition in New World Primates. J Virol. 2005, 79(23): | 4988-| 5003.
Publish with Biomed Central and every scientist can read your work free of charge

"BioMed Central will be the most significant development for disseminating the results of biomedical research in our lifetime. "

Sir Paul Nurse, Cancer Research UK

Your research papers will be:

- available free of charge to the entire biomedical community

- peer reviewed and published immediately upon acceptance

- cited in PubMed and archived on PubMed Central

- yours - you keep the copyright
BioMedcentral 